\title{
Portable Heart Rate Detector Based on Photoplethysmography with Android Programmable Devices for Ubiquitous Health Monitoring System
}

\author{
Chi Kin Lao, U Kin Che, Wei Chen, Sio Hang Pun, Peng Un Mak, Feng Wan, and Mang I Vai
}

\begin{abstract}
In this paper, a miniature portable heart rate detector system is implemented by modern hardware ICs and simple sensor circuit with software executable on both PC and Android platform. The biosignal is first extracted via photoplethysmography (PPG) principle into electric signal. Then a microprocessor is used to covert biosignal from analog to digital format, suitably for feeding into an RF module (nRF24L01 for RF transmission). On the receiver end, the computer and/or smart phone can analyze the data using a robust algorithm that can detect peaks of the PPG waveform, hence to calculate the heart rate. Some application software running on Windows and Android phone have been developed to display heart rate information and time domain waveform to users for health care monitoring. In the future, pure Bluetooth technology will be used for wireless personal communications instead of RF modules. At the same time, the data can be sent to computer console using existing available networks (3G, 4G, WiFi, etc.) for health database logging purpose.
\end{abstract}

Keywords-Heart rate detector, Android application, healthcare monitoring, Photoplethysmography (PPG), peak detection algorithm, wearable/portable device.

\section{INTRODUCTION}

Because of the rapid medical development of our modern societies, the health care system becomes much more mature and professional. Migrating regular mature in-clinic/inhospital health system to individual wearable monitoring systems for chronic disease becomes popular. This trend will go on in forthcoming years as the average number of elderly has continued to occupy large portions worldwide. In order to decentralize the current burden of public health system and promote the popularity of routine health self-check, many techniques in Biomedical Engineering have been developed for making faster and more accurate pre-diagnoses with easeof-use.

The traditional devices are quite bulky, so the patients usually need to go hospital/clinic every time whenever health

This work was supported in part by the Science and Technology Development Fund of Macau under Grant 063/2009/A, Grant 024/2009/A1, and Grant 036/2009/A; the Research Committee of the University of Macau under Grant UL12/09-Y1/EEE/VMI01/FST, Grant RG077/09-10S/VMI/FST, Gran RG072/09-10S/MPU/FST, and Grant MYRG139(Y1-L2)-FST11-WF.

All authors are with Department of Electrical and Computer Engineering, Faculty of Science and Technology, University of Macau, Macau SAR China (phone: 853-8397-4276; fax: 853-8397-4275; Corresponding email fstpum@umac.mo). Mang I Vai and Sio Hang Pun are also with the State Key Laboratory of Analog and Mixed-Signal VLSI, University of Macau, Macau SAR, China) and Peng Un Mak is currently on leave to the Anschutz Medical Campus, University of Colorado, USA

Manuscript received Oct. 28, 2012; revised December 16, 2012. checks are conducted even for slight discomfort. This is quite inconvenient, especially for the patients with chronic diseases and/or with moving difficulty. Although some small mobile devices [1][2] have been developed recently, they are still equipped with some unwieldy cables, which lead to some inconvenience. Lately, biomedical devices using wireless techniques are developed, and their sizes become much smaller than those before.

To address the health problem mostly related to the general public, small devices for the cardiovascular diseases (CVDs) can provide timely $\&$ immediately supports for the most patients at home. In according to the World Health Organization, CVDs are the first cause of death, equivalently $30 \%$ of global deaths in 2008. This figure is estimated to rise up to about 25 million of deaths by 2030 [3]. Despite of devoting efforts in prevention of CVDs, we should also prepare for easing the increasing pressure for the public health system.

Electrocardiography (ECG) monitoring has been a standard norm procedure for CVDs diagnosis and the need to develop a miniaturized device for self-assessment and monitoring of the CVDs patient is important. By measuring the Heart Rate Variability (HRV), the status of the heart activities can be estimated. However, measuring the ECG of the patient is not easy for untrained personnel, not mentioning long-term monitoring without significant interference in the daily life. Although it is less prominent than that from ECG [4], HRV from PPG can be still an acceptable compromise for non-professional and daily monitoring. PPG would be more acceptable for general public because of single detection location (usually at fingertip or ear lobe) and no gel electrode needed. It is one of non-invasive methods for measuring the amount of the blood volume changes inside the blood vessel [5]. In general, PPG sensing device is easy to position for long term monitoring purpose and can monitor several CVD related parameters such as heart rate, blood oxygen level, respiration, etc.

In this paper, a portable biomedical detector prototype that monitors heart rate and potentially measures blood oxygen level in the future using PPG is designed and implemented, as well as a robust PPG waveform detection algorithm is proposed. To minimize the size of the system, hardware devices were carefully chosen with multiple functions on the same chip. Also wireless communication is realized by small size RF modules and leave further analyses for computers/PDAs. The next Section gives the system overview. Section III introduces various subsystems/building blocks \& prototype results for our implementation. Section [IV] explains the technical 
details about the adopted peak detection algorithm. Prototype results and conclusions are given in Section $\mathrm{V}$ and future improvement suggestion can be found in the end.

\section{SySTEM OVERVIEW}

The overall design block diagram of our PPG heart rate detector is shown in Fig. 1. In the current design, the system is divided into two main parts: PPG detector and Human Interface. The PPG detector, as shown in the upper portion of Fig. 1, consists of a battery-powered standalone device consisting of a special designed optical sensor for PPG detection, a conditioning circuit for noise reduction \& anti-aliasing, a MCU for digitizing the PPG signal \& preparing the data for transmission, and a RF transmitter for connection to the server computer.

The block diagram of the Human Interface, as shown in the lower part of Fig. 1, consists of a RF receiver, a server computer and an Android ready mobile phone. The server computer communicates with the PPG detector via the RF receiver. In the same time, the PPG data is stored for in-depth analysis for the purposes of diagnosis and treatment planning in the future. In the meanwhile, related information is sent to a smartphone for user's concern wirelessly. In the next Section, the function of each component will be briefly discussed.
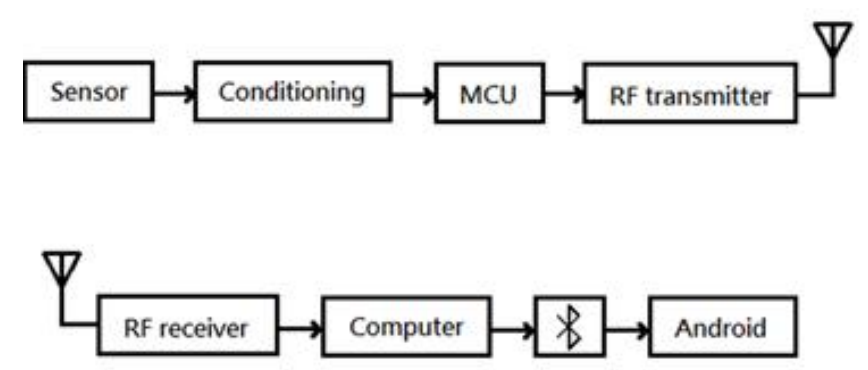

Fig. 1. Block diagram of the PPG heart rate detector and Human interface

\section{FUNCTIONAL PARTS \& PROTOTYPE RESULT}

In this section, the role and characteristics of the each module within the system are introduced and described.

\section{A. Wearable Sensor}

To facilitate plethysmography measurement, three sensing mechanisms are commonly used, namely, volume displacement plethysmography, impedance plethysmography, and photoplethysmography [6]. The photoplethysmography is preferred in our design because measurement can be performed on fingertip without precise positioning. Additionally, the design can easily upgrade to blood oxygen saturation measurement. Photoplethysmography (PPG) is based on plethysmography and photovoltaic technique, as displayed in Fig. 2(a). Every time when blood pumps to periphery (ejection phase), blood vessels expand due to the blood pressure from the heart, a pulse will be generated. And every time when the blood flows back (diastolic filling phase), another pulse follows. So the PPG signal will be the superposition of the pumping pulse

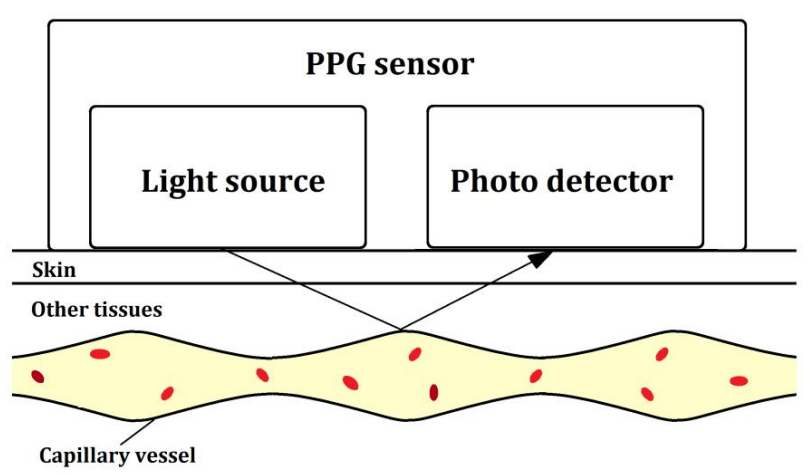

(a)

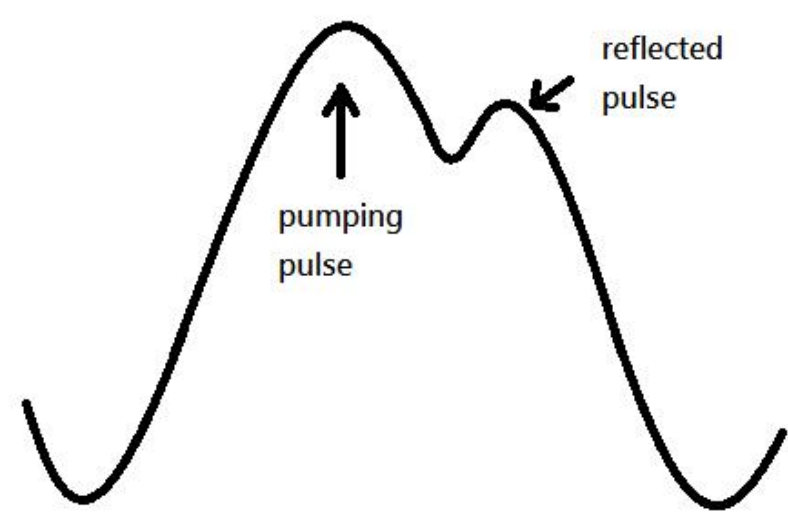

(b)

Fig. 2. (a) Basic PPG technique; (b) Sample PPG waveform

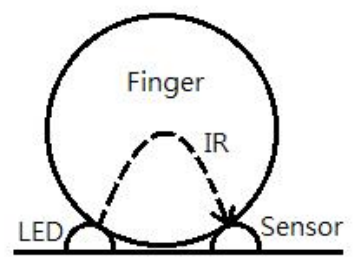

(a)

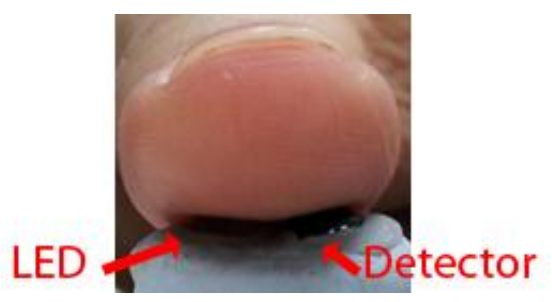

(b)

Fig. 3. (a) Illustration of PPG sensor schematic; (b) physical case during signal extraction

and the reflected wave, as shown in Fig. 2(b)

Fig. 3 depicts our conceptual diagram of PPG measurement on fingertip. An infra-red LED and a detector were placed beneath the fingertip of the patient for measuring the change of blood volume by the activities of the heart. The volume change of the blood within the artery will directly affect the scattering light 
received by the photo-detector and thus, a simple circuitry, as shown in Fig. 4, is used to generate the electric pulsation related to the plethysmography. A sample output of the sensor in both time and frequency domains is shown in Fig. 5. One can observe the heart rate is located around 1-2 $\mathrm{Hz}$ while the power line $50 \mathrm{~Hz}$ is strongly presented.

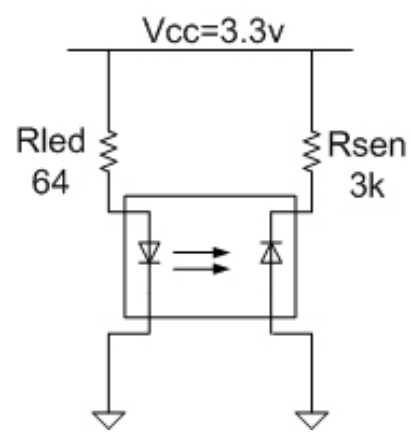

Fig. 4. Block diagram of the PPG heart rate detector

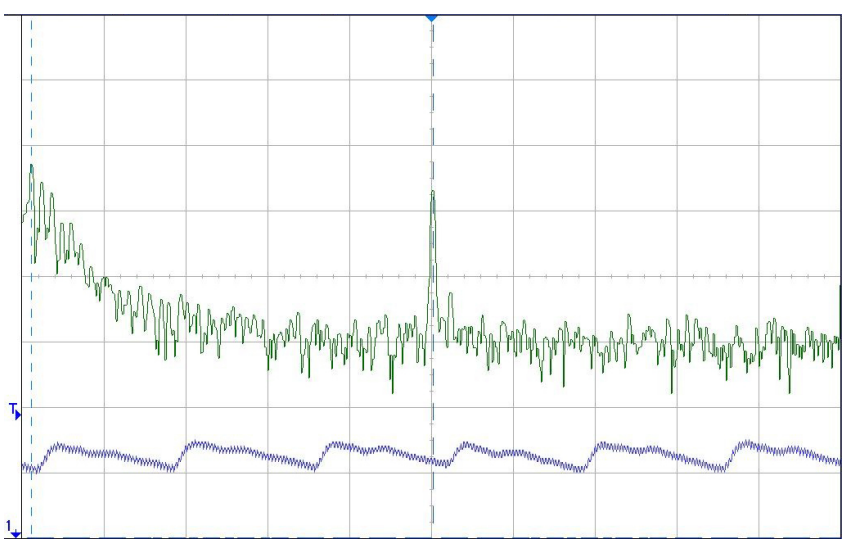

Fig. 5. Acquired Electric pulsation of the plethysmography from sensor (Purple colour: Time domain \& its FFT in green colour)

\section{B. Signal Conditioner}

As one can observe the PPG signal in Fig. 5, the raw signal from the sensor is quite weak $(8 \mathrm{mVp}$-p) and is contaminated with noise (especially $50 \mathrm{~Hz}$ power-line) on top of a DC component. Therefore, a high pass filter with stop corner frequency of $0.5 \mathrm{~Hz}$, as shown in Fig. 6, is applied.

Additionally, an operation amplifier (AD626), which has

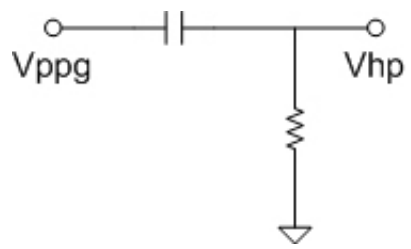

Fig. 6. Circuit to filter out the DC components of the PPG signal

maximum $100 \mathrm{~V} / \mathrm{V}$ voltages gain and intrinsic structure for adding low pass filter (corner frequency set to $34 \mathrm{~Hz}$ ), was used to eliminate the noise component in the signal and boosted the signal for the A/D converter in the next stage. The schematic of the pre-amplifier can be found in Fig. 7(a) while its frequency response is shown in Fig. 7(b) The corner frequencies of high pass and low pass filters in this signal conditioning block setting satisfied the ordinary PPG signal with frequency in several $\mathrm{Hz}$ [7], and its bandwidth of the PPG signal is $0-30 \mathrm{~Hz}$ [8].

Fig. 8 displays the processed PPG signal before entering

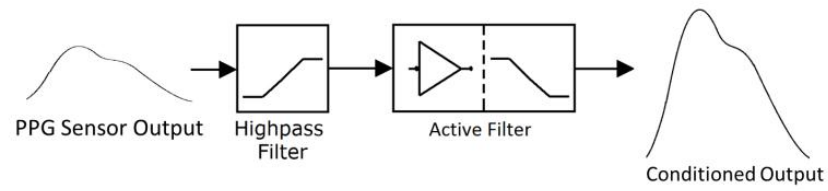

(a)

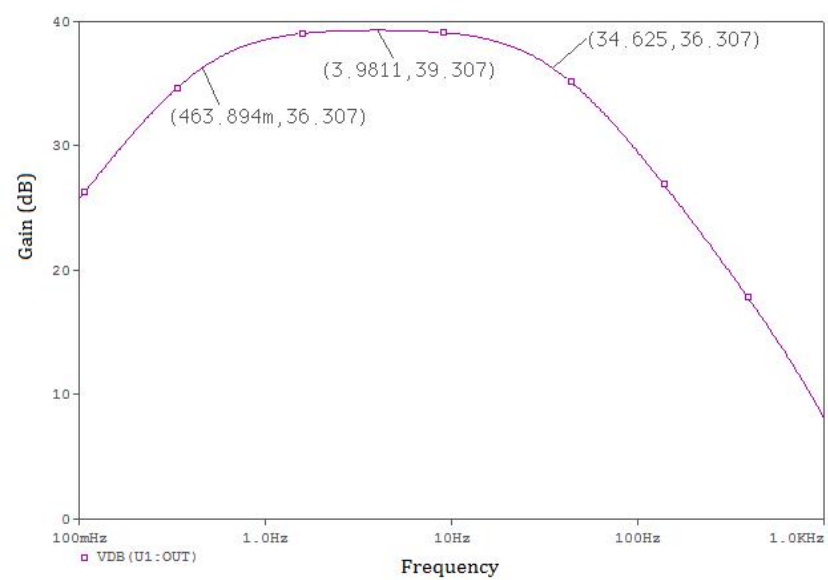

(b)

Fig. 7. (a) Schematic of the pre-conditioning module; (b) Its frequency response

the next stage of microprocessor. The PPG signal and its characteristics can be clearly identified.

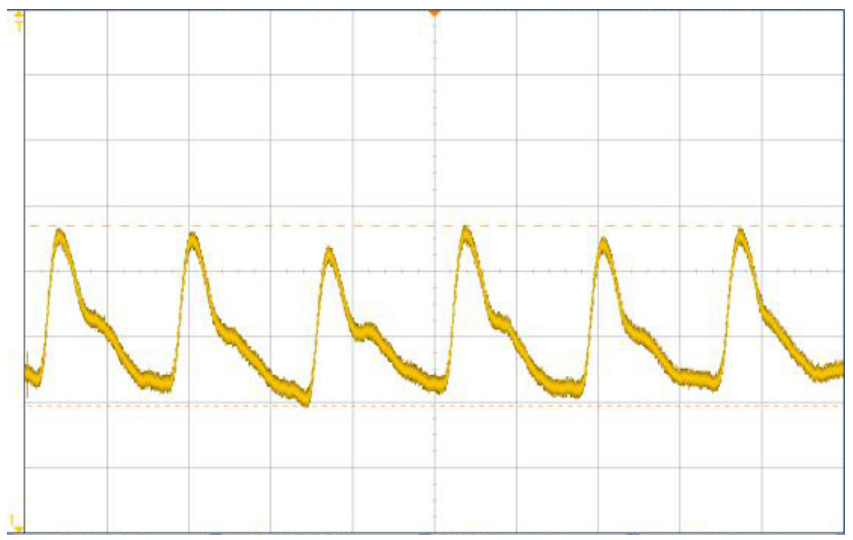

Fig. 8. Sample Conditioned PPG signal

\section{Microprocessor}

To build a wearable PPG device with extended battery life, we select the C8051F930, which belongs to an 8051 family microprocessor with built-in 10-bit ADC, $90 \mu \mathrm{A}$ supply 
current, and small form factor $9 \times 9 \times 1.6 \mathrm{~mm}^{3}$. The sampling frequency of the $\mathrm{ADC}$ is set to be $250 \mathrm{~Hz}$.

\section{RF Transceiver}

nRF24L01 [9] is used to wirelessly transmit the PPG signal to the server computer or equivalent. It is chosen because of its small size of around $4 \times 4 \mathrm{~mm}^{2}$ and can be operated through a standard Serial Peripheral Interface (SPI). The carrier frequency is ISM frequency band around $2.4 \mathrm{GHz}$ and operates up to $1 \mathrm{Mbps}$ data rate. The supply voltage is $3.3 \mathrm{~V}$ from lithium ion battery of $70 \mathrm{mAh}$ capacity.

\section{E. Finger Clip Housing}

In order to accommodate all the hardware of aforementioned subsystems, we use a 3-D printer, Fortus 400mc model [10] to print our finger chip housing. This can allow us to flexibly minimize the mechanical housing of our clip in ergonomic consideration; and at the same time, to provide hidden room for various electronic building blocks. Fig. 9(a) shows our home-made finger clip housing outlook while Fig. 9(b) \& 9(c) display various electronics components in assembly. The initial prototype size is around $40 \times 30 \times 20 \mathrm{~mm}^{3}$ and could be smaller in later version.

\section{F. Windows Application}

As Android phones and iPhones are the most popular phones nowadays and possibly in near future, we would like to select one of them to develop our PPG application. Finally we choose Android because development on iOS has much more limitations in comparison to those on Android, which belongs to Open-Source. However, apps that are not from the Apple Apps Store cannot be installed to a standard iPhone. For an Android phone, we just need to develop our app to be a signed app, then our app can be installed on the phone and start the testing. A Windows application written in Visual Basic is developed as a server for processing the PPG signal. In this stage, a new peak detection algorithm for heart rate calculation is designed, which is fast enough to satisfy the realtime application on the smartphone and somewhat adaptive and predictive to achieve its robustness. The algorithm will be discussed in details in the next section.

The whole digital processing including the peak detection and procedure of heart rate calculation is shown in Fig. 10. The mini-system continuously receives the PPG from the wearable sensors wirelessly, processes the raw PPG signal, stores for future reference, and retransmits the PPG signal \& information to a smartphone app via Bluetooth. In addition to these basic functions, the server computer can also display the PPG signal in real time (as shown in Fig. 11). One can observe that Fig. 11 basically retains most information carried by Fig. 8. Hence, Heart Rate can be readily obtained.

\section{G. Android App}

In order to display the PPG signal in smartphone devices, an Android apps was developed. The Bluetooth APIs of Android

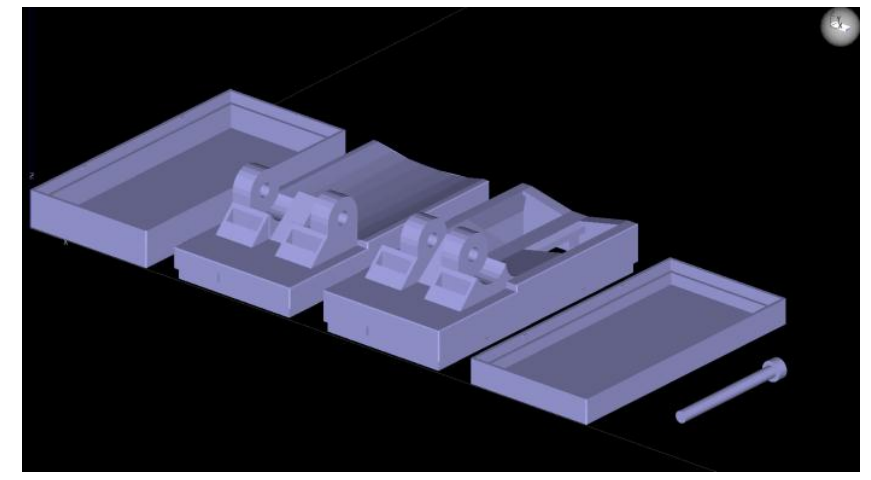

(a)
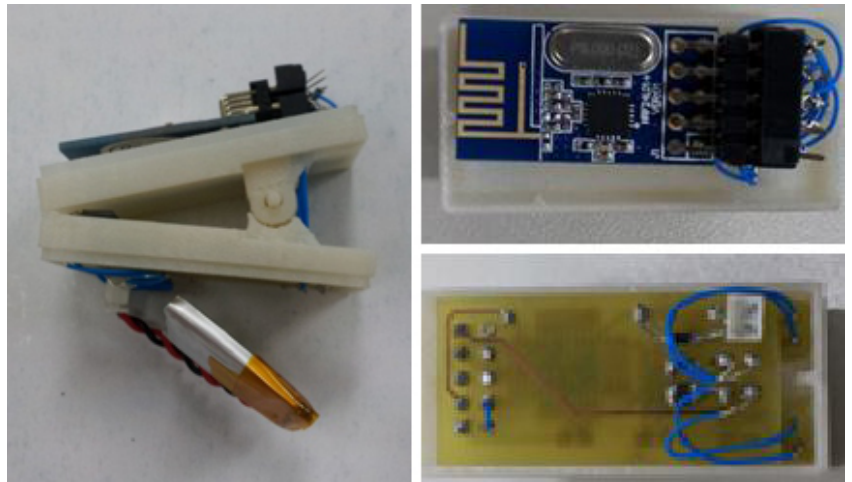

(b)

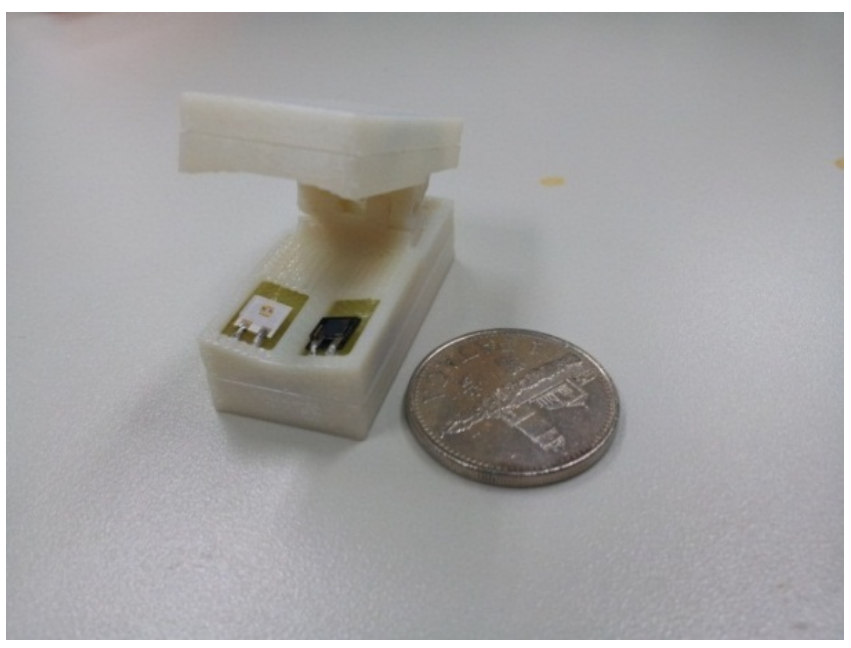

(c)

Fig. 9. (a) Finger clip housing; (b) microprocessor, and RF module; (c) Sensor circuit with 1 Macau Pataca Coin

supports the cable replacement protocol RFCOMM which provides serial port (RS-232) emulation. By using the serial port emulation mode, the PPG data with necessary information can be easily delivered to the smartphone apps without excess computing consumption. The main technique of this kind of communication is to establish a virtual serial port while pairing with the Android phone using Fig. 10 algorithm. Finally, an open source pure Java API, AndroidPlot, was used to create dynamic PPG chart. Its library was added to our Android application, then the plot view can be added to the layout main file and the corresponding program which is based on 


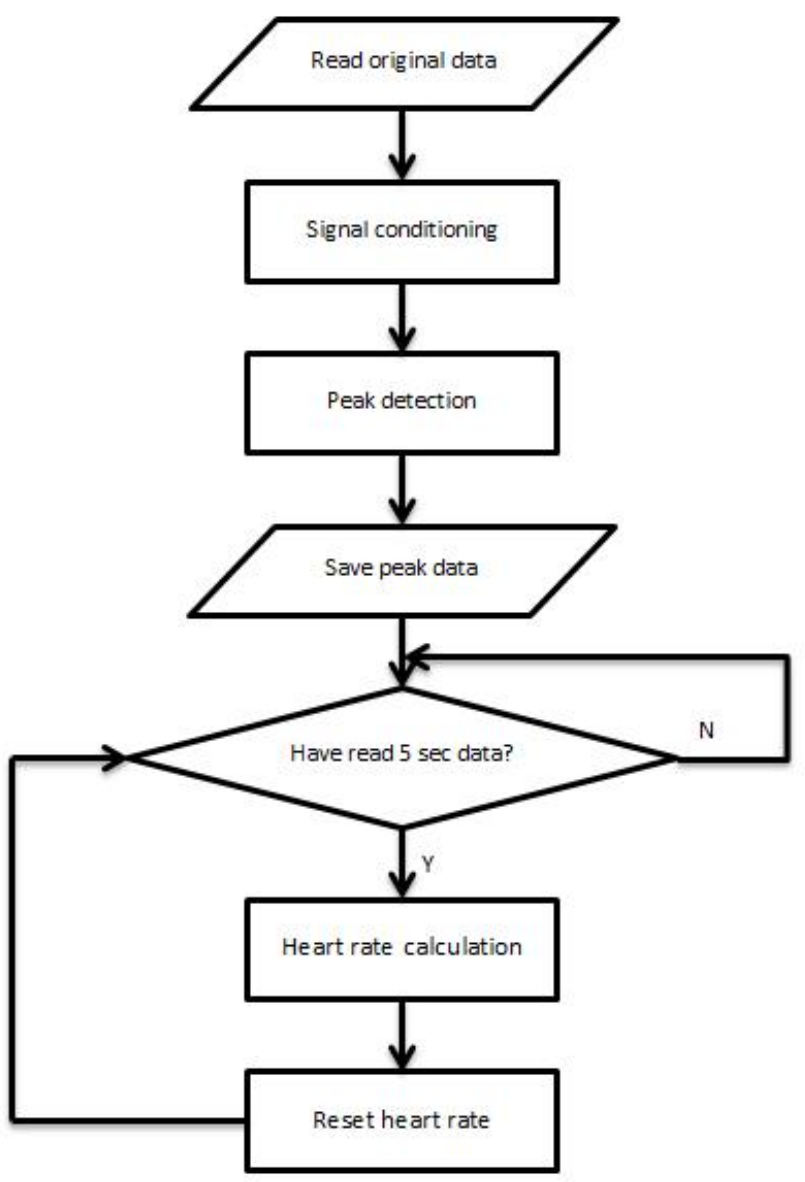

Fig. 10. Flowchart of our digital processing

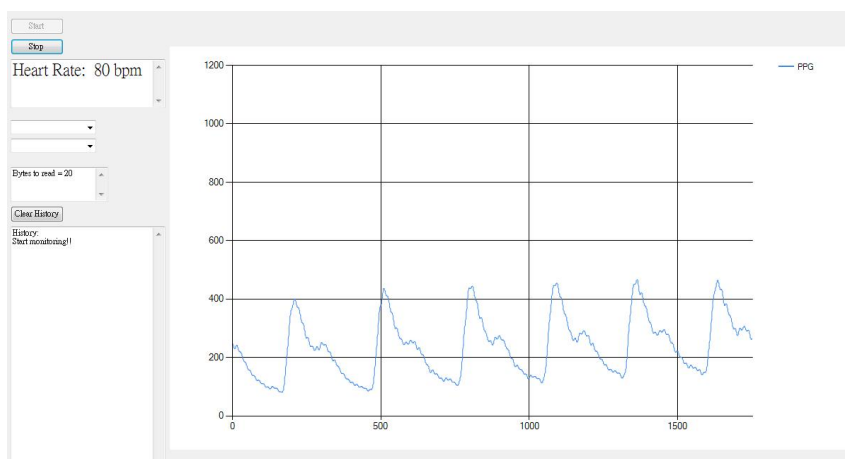

Fig. 11. Sample PPG plot from the server computer

the AndroidPlot example "Orientation Sensor" is added to the main activity file. When there is new PPG data reading, the PPG plot will be updated, as shown in Fig. 12 below.

\section{PPG SIGNAL PROCESSING ALGORITHM DESIGN}

Admittedly, PPG waveform has a lesser complicated morphology than other physiological signals, such as EEG and ECG and etc. However, there always exist high-frequency noises as well as the low-frequency baseline drift, derived from either the measurement itself or respiration and motion artifacts. Recently, there are several PPG signal processing algorithms proposed in the recent years, including adaptive

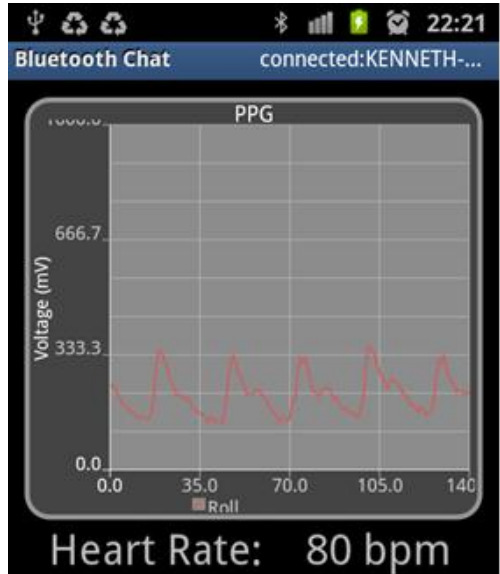

Fig. 12. Sample PPG plot in a Smart Phone

threshold method [11], cubic spline interpolation [12], local maxima method [13][14]. They almost contain two parts, namely the signal pre-processing/conditioning and peak detection or feature extraction.

Likewise, our algorithm for PPG signal processing also primarily consists of two parts: signal conditioning [13] and peak detection [11]. The algorithm was previously implemented in MATLAB R2011a (The Mathworks, Inc.), and then transformed into Visual Basic 2010 Express which is the platform of the whole system. For convenience, subsequent examples and results will be demonstrated in the MATLAB.

\section{A. Signal Conditioning}

For high-frequency noises cancellation and baseline drift removal, various types of digital filtering methods prove to be effective. Besides, discrete wavelet transform [15] can also act as a denoiser to smooth the waveform. Our signal preprocessing mainly contains two steps: high-pass FilterDxN [16] for real-time baseline drift removal and low-pass moving average filter [17] to smooth the waveform.

The proposed FilterDxN was initially named after the two parameters adjusted in the design of it, namely $N$ for the number of the averaged signal samples and $D$ for the distance between them. Typically, the filter can be expressed as:

$$
y[i]=x[i]-\frac{1}{N} \sum_{j=-(N-1) / 2}^{(N-1) / 2} x[i+j D]
$$

where $x[i]$ is the input signal and $y[i]$ is the output signal. In order to avoid the phase shifting, $N$ should be selected as an odd number. It can be perceived that we can simply adjust $D$ and $N$ in accordance with the performance we expect. The implementation of the filter is relatively easy, compared with the complex and time-consuming frequency and time domain conversion. For the consideration of the actual performance, we manually conducted averaging over a group of 25 samples with a distance of 15 samples between each other, thereby realizing a comb filter to reduce the baseline drift.

As the name implies, the moving average filter operates by averaging a number of consecutive samples from the input 
signal to replace corresponding samples in the output signal:

$$
y[i]=\frac{1}{N} \sum_{j=-(N-1) / 2}^{(N-1) / 2} x[i+j]
$$

Again we can see that $N$ should be an odd number just for symmetrical consideration. In our system (sampling frequency of $250 \mathrm{~Hz}$ ), we chose $N$ as 21 to realize a low-pass filter in removing high-frequency noises.

Fig. 13 displays the original PPG signal as well as the results of signal conditioning (baseline removal and noise cancellation), respectively. From the figure, it can be seen that the signal conditioning removes baseline drift and noises, resulting PPG with only very little dc component. This would be more convenient for us to utilize the information inside the amplitude in the peak detection. However, the tradeoff of this single conditioning is to have minor loss of samples at both ends due to the moving average used in both detrending and denoising.

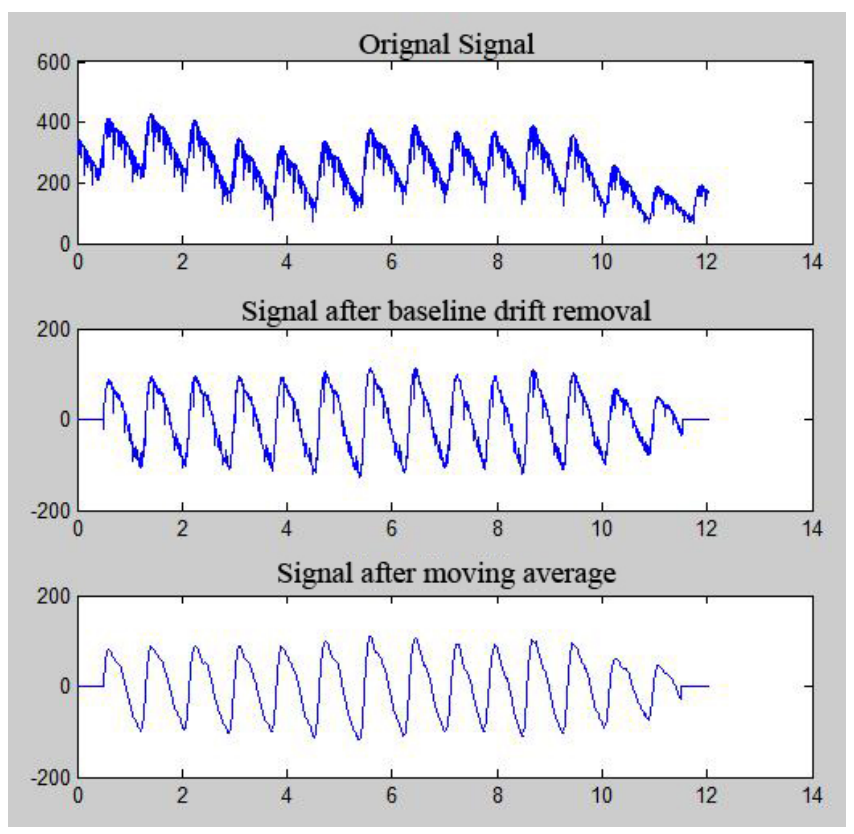

Fig. 13. Original PPG signal and results of detrending and denoising

\section{B. Peak Detection}

Actually, the original ideal of our peak detection method comes from one adaptive threshold method for the peak detection of PPG waveform [11]. However, we have modified or even improved it by changing the adaptive threshold rule and adding some new considerations to deal with certain extreme waveform conditions. We call it the sliding and climbing (S\&D) peak detection method because the peak detection procedure is quite similar to the repetitive process of sliding downhill from one peak and then climbing uphill to the next peak. The overall flow diagram of the S\&D method is described in Fig. 14

In order to better clarify the procedure of the S\&D peak detection method, a typical example is graphically illustrated in Fig. 15. Other more complicated or tough conditions will be shown later to demonstrate the advancement and robustness of our peak detection method.

In the process of S\&D peak detection, the value of threshold is decreased with an adaptive slope during the period when the threshold is higher than the PPG signal. Like simple envelop detector, when meeting the signal, the threshold will merely follow or track the signal until it detects a new peak. At the new peak, the slope is updated according to the amplitude of current peak and next potential one, and starts another sliding and climbing process. Former procedures are repeated till the end of the signal.

In adaptive thresholding, the current slope is modified by the amplitude of the latest peak and amplitude difference between the latest peak and next potential one. The modified slope can be expressed by (3).

$$
\text { slope }_{i}=c_{r} V_{i}+f_{r}\left(V_{i}-V_{p}\right)
$$

where slope $_{i}$ is the descending slope of the $i^{\text {th }}$ peak, $c_{r}$ and $f_{r}$ are current and future factor weights, and finally $V_{i}$ and $V_{p}$ stand for the amplitudes of current peak and next potential one. In our system, $c_{r}$ and $f_{r}$ are empirically selected as -0.6 and -1.0 , respectively. Flexibility of this method is to allow adjusting these two parameters to seek for a better performance in the application.

After the signal pre-processing, most noises and baseline drift

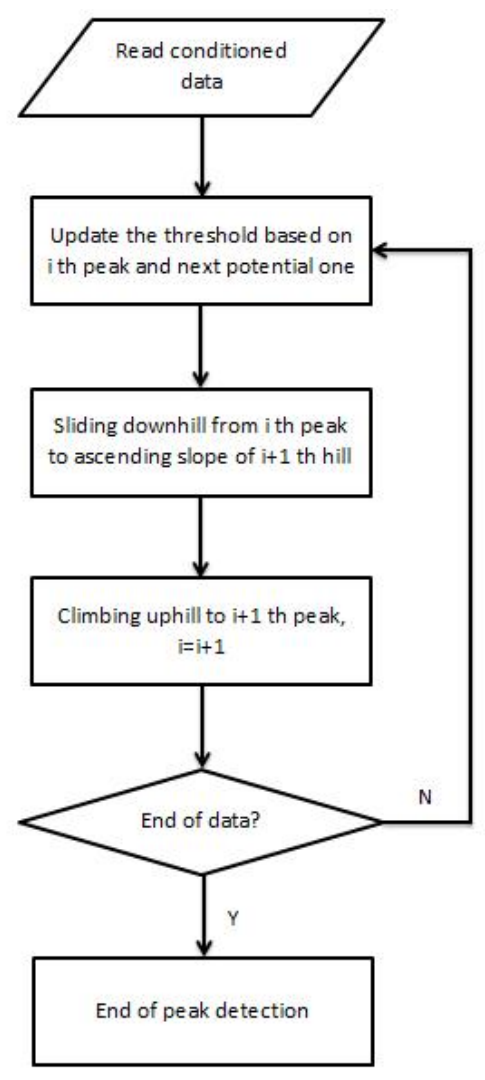

Fig. 14. Peak detection flow diagram of the S\&D method

should have been eliminated. However, some rigid ripples or local maxima points usually exist to hinder the peak detection. Fig. 16(a) and Fig. 16(b) illustrate the situation and the 


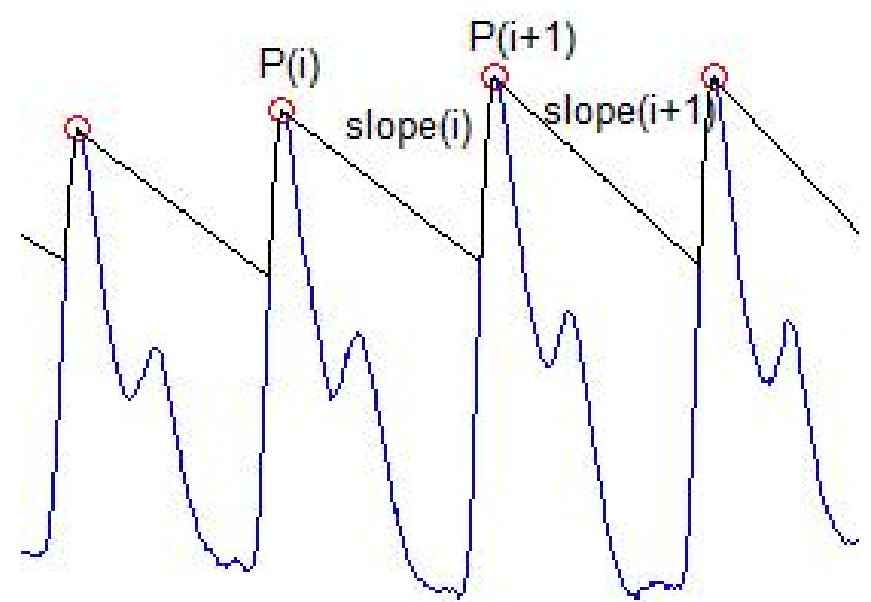

Fig. 15. An illustration of typical procedure in S\&D peak detection

associate solution to conquer it.

To overcome the ripples on ascending slope or near the peak,

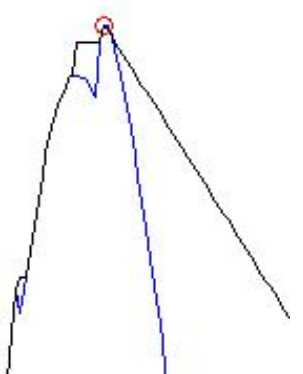

(a)

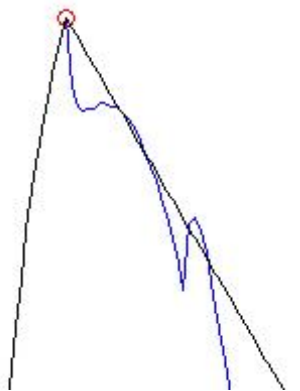

(b)
Fig. 16. (a) Ripples on the ascending slope and around the peak; (b) Ripples on the descending slope

when meeting a local maxima point, instead of regarding it as a new peak, our peak detection method will conduct a scanning action over a tiny interval exactly after the detected local maxima point. If there is no sample that has a larger value there, then the newly found point will be considered as a true peak. Otherwise, the threshold will skip the small ditch and continue to climb.

For the ripples on the descending slope, which the threshold line has a chance to meet before the ascending slope of the next hill, our S\&D detection method applies a similar measure to the one used in another adaptive threshold method [11] A criterion based on the previous average pulse interval is adopted to judge whether the threshold has arrived at the ascending slope of the next hill. If the distance between the crossing point and the latest peak is larger than 0.6 times of previous average peak interval, the threshold will stop and follow the signal. On the contrary, it will keep sliding down. This criterion also takes effect when coping with high reflected wave peak as a result of aging.

Generally, after baseline removal, there should not be any large sudden variation in amplitude between two successive waves. However, in order to test our peak detection method, we artificially modify one period of actual signal to generate the extreme situation. To our satisfaction, the detection method successfully gets through the test, which is shown in Fig. 17 Since the adaptive slope contains both the current and future

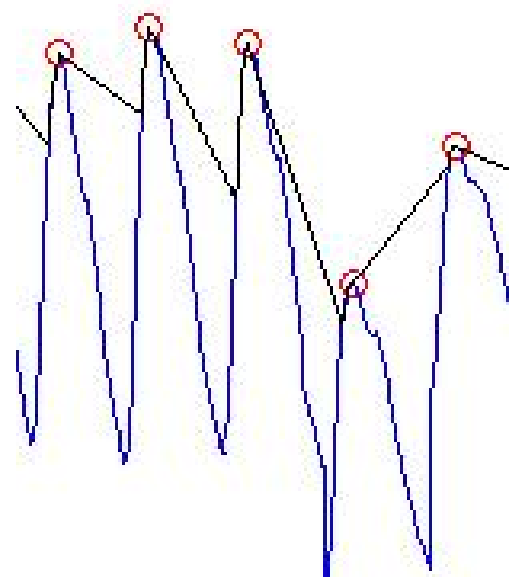

Fig. 17. Solution of large sudden amplitude variation

information about amplitude, our detection is sensitive to the variation of amplitude, thus ensuring the validity and robustness of our peak detection method. Finally, the result of signal conditioning and peak detection for a sample PPG signal is shown in Fig. 18(a) and Fig. 18(b) respectively.

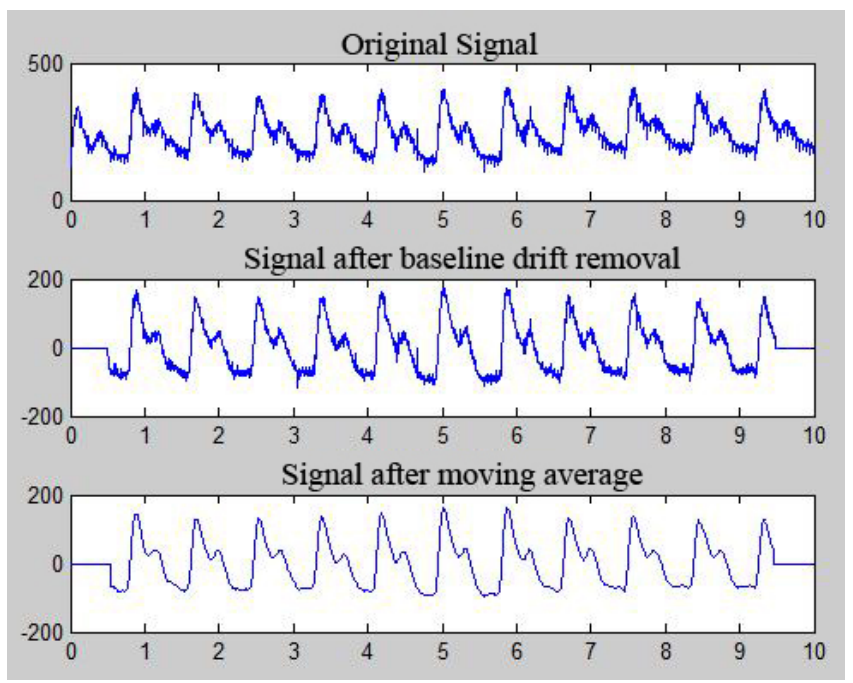

(a)

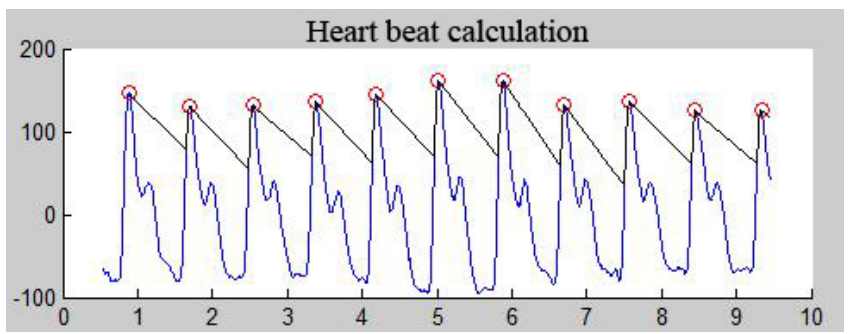

(b)

Fig. 18. (a) Result of signal conditioning for a sample PPG signal; (b) Result of peak detection of the signal 


\section{CONCLUSIONS}

In this work, we have successfully developed a portable heart rate detector for monitoring CVDs patients so as to address the health problem mostly related to the general public. This heart rate detector uses PPG to calculate the HRV of the patient in order to assess the abnormal symptoms such as arrhythmia, coronary artery diseases, etc. Because of the light weight PPG heart rate detector housing in a tailor made finger clip, this system is compatible with the popular Android smart phone so that the waveform of the PPG, realtime heart rate \& HRV can be displayed to the user without additional hardware. Furthermore, from our design, the system can provide initial information regarding to the health status of the CVDs patients, and index for pre-diagnosis of various cardiovascular symptoms as well.

At the same time, a robust algorithm for PPG waveform processing (signal conditioning and peak detection) is designed. Without relatively complicated frequency analysis and wavelet decomposition in signal pre-processing step, this adaptive algorithm is fast enough to realize the real-time health care monitoring application with validations from experiments. Therefore, the algorithm itself should have a good application prospect.

\section{FUTURE DEVELOPMENTS}

In the future development, we would test more subjects and compare our result with the standard Holter ECG medical equipment for accuracy evaluation. On the other hand, to increase the versatility of heart rate during exercise, we shall add acceleration sensor to remove the motion artifacts. Also, we would like to extend the program in Android, so that it can transmit the PPG data to the Internet via 3/4G networks. To the final end, we can integrate all electronic devices in transmitter module into a single chip, like SOC, so that the device can be extremely small or even implantable into human for signal extraction.

\section{REFERENCES}

[1] P. Wei, R. Guo, J. Zhang, and Y. Zhang, "A new wristband wearable sensor using adaptive reduction filter to reduce motion artifact," in Proceedings of the 5th International Conference on Information Technology and Application in Biomedicine, in conjunction with The 2nd International Symposium \& Summer School on Biomedical and Health Engineering.

[2] K. I. Wong, "Design and experimentation of wearable body sensors," pp. 273-288, Rapid Prototyping Technology - Principles and Functional Requirements, Dr. M. Hoque (Ed.), Intech, 2011.

[3] W. H. Organization, "Cardiovascular diseases (cvds)," Fact sheet No. 317, Sep. 2012.

[4] F. C. Chang, C. K. Chang, C. C. Chiu, S. F. Hsu, and Y. D. Lin "Variations of hrv analysis in different approaches," Computers in Cardiology 2007, Vols 1 and 2, pp. 17-20, 2007. Bja59 Times Cited:0 Cited References Count:14 Computers in Cardiology.

[5] J. Allen, "Photoplethysmography and its application in clinical physiological measurement," Physiological Measurement, vol. 28, no. 3, pp. R1-R39, 2007. 150JQ Times Cited:141 Cited References Count:269.

[6] R. B. Northrop, Plethysmography, p. 525 p. Biomedical engineering series, Boca Raton: CRC Press, 2002.

[7] J. S. Rhee, Finger Photoplethysmographic Sensors for Vital Sign Monitoring. PhD dissertation, Massachusetts Institute of Technology, 2000.

[8] M. Nitzan, A. Babchenko, and B. Khanokh, "Very low frequency variability in arterial blood pressure and blood volume pulse," Med. Biol. Eng. Comput., vol. 37, no. 1, pp. 54-58, 1999.
[9] “www.nordicsemi.com/eng/products/2.4ghz-rf/nrf24101," Last accessed on Feb. 2012.

[10] "http://www.fortus.com/products/fortus-400mc.aspx," Last accessed on Feb. 2012.

[11] H. S. Shin, C. Lee, and M. Lee, "Adaptive threshold method for the peak detection of photoplethysmographic waveform," Computers in Biology and Medicine, vol. 39, no. 12, pp. 1145-1152, 2009.

[12] W. Chen, S. Lei, L. Guo, Y. Chen, and M. Pan, "Study on conditioning and feature extraction algorithm of photoplethysmography signal for physiological parameters detection," in Image and Signal Processing (CISP), 2011 4th International Congress on, vol. 4, pp. 2194-2197.

[13] B. Nenova and I. Iliev, "An automated algorithm for fast pulse wave detection," International Journal Bioantomation, vol. 14, no. 3, pp. 203216, 2010.

[14] S. Lu, H. Zhao, K. Ju, K. Shin, M. Lee, K. Shelley, and K. H. Chon, "Can photoplethysmography variability serve as an alternative approach to obtain heart rate variability information?," Journal Of Clinical Monitoring And Computing, vol. 22, no. 1, pp. 23-9, 2008.

[15] A. K. Bhoi, S. Sarkar, P. Mishra, and G. Savita, "Pre-processing of ppg signal with performance based methods," INTERNATIONAL JOURNAL OF COMPUTER APPLICATION, vol. 4, no. 2, pp. 251-256, 2012.

[16] I. T. Iliev, "Combined high-pass and power-line interference rejecter filter for ecg signal processing," Proceedings of the Technical University - Sofia, Bugaria, vol. 58, no. 2, pp. 7-13.

[17] S. S. W., The Scientist and Engineers Guide to Digital Signal Processing. San Diego, CA,: California Technical Publishing, 2nd ed., 1999.

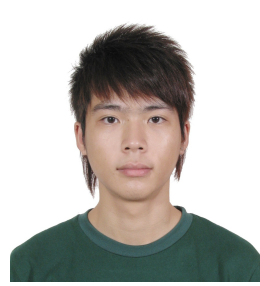

Chi Kin Lao, received the Bachelor degree in Electrical and Electronics Engineering, University of Macau, Macau, 2012.

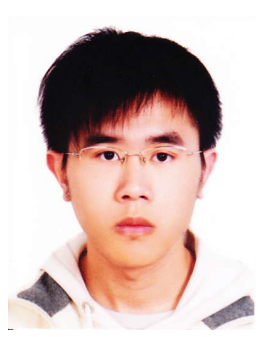

U Kin Che, received the Bachelor degree in Electrical and Electronics Engineering, University of Macau, Macau, 2012.

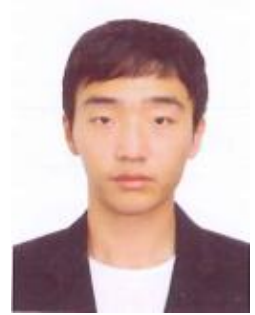

Wei Chen, received the Bachelor degree in Automation from Zhejiang University, China, 2012. Now he is pursuing his master degree in Electrical and Computer Engineering from University of Macau and is a student member of IEEE. His current research interests are Digital Signal/Image Processing, Intelligent Control and Computational Intelligence. 
Sio Hang Pun, received the Master degree in Computer and Electrical program from the University of Porto, Portugal, 1999. He also received the $\mathbf{P h}$. D Degree in the Electrical and Electronics Engineering from the University of Macau, Macau, 2011. Since 2000, he has performed research in the areas of biomedical engineering. His research interests are Bio-electronic circuits, Intra-Body Communications, and Bioelectromagnetism. He is currently an Assistant Professor of the State Key Laboratory of Analog and Mixed-Signal VLSI, University of Macau, Macau.

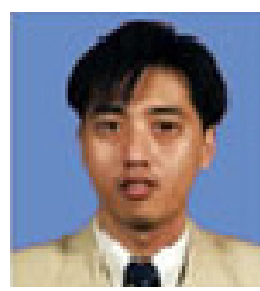

Peng Un Mak received his BSc. from National Taiwan Univ., MSc. \& PhD from Michigan State Univ., all in Electrical Eng. Since, 1997, he has joined University of Macau as an assistant professor in Dept. of Electrical \& Computer Engineering. His current research interests are Bio-electromagnetism, Intra-Body Communication \& bio-electric signals acquisition. He has authored/coauthored over 100 peer-reviewed technical publications (journal, book-chapter, conference proceedings, and etc.).

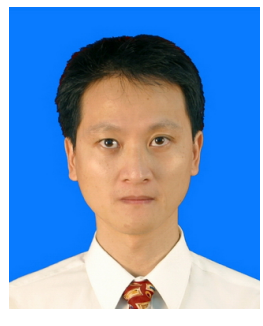

Feng Wan received the $\mathrm{PhD}$ degree in Electrical and Electronic Engineering from the Hong Kong University of Science and Technology, Hong Kong. He is currently an Assistant Professor in the Department of Electrical and Computer Engineering, Faculty of Science and Technology, University of Macau, Macau. His research interests include biomedical signal processing, brain-computer interfaces, neurofeedback training, computational intelligence and intelligent control.

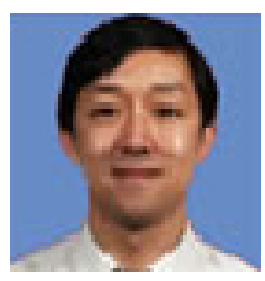

Mang I Vai, received the Ph.D. degree in Electrical and Electronics Engineering from the University of Macau, Macau SAR, China in 2002. Since 1984, he has performed research in the areas of digital signal processing and embedded systems. He is now an Associate Professor of the Department of Electrical and Computer Engineering, Faculty of Science and Technology and the State Key Laboratory of Analog and MixedSignal VLSI, University of Macau. 